\title{
Cause-specific mortality in individuals with severe alpha I-antitrypsin deficiency in comparison with the general population in Sweden
}

This article was published in the following Dove Press journal:

International Journal of COPD

26 July 2016

Number of times this article has been viewed

\section{Hanan A Tanash' \\ Magnus Ekström² \\ Philippe Wagner ${ }^{3}$ \\ Eeva Piitulainen'}

'Department of Respiratory Medicine, Skåne University Hospital, ${ }^{2}$ Department of Respiratory Medicine, Blekinge Hospital Karlskrona, Lund University, Lund, ${ }^{3}$ Centre for Clinical Research, Västmanland, Uppsala University, Uppsala, Sweden
Correspondence: Hanan A Tanash Department of Respiratory Medicine, Skåne University Hospital, Inga Marie Nilssons gata 46, S-205 02 Malmö, Sweden

Tel +4640331000

Fax +46 40336225

Email hanan.tanash@med.lu.se
Background: Severe alpha 1-antitrypsin deficiency (PiZZ) predisposes to morbidity and mortality due to early-onset emphysema and liver disease. The risk of death from other causes, including cardiovascular disease and cancer, has not been well investigated. We aimed to analyze cause-specific mortality in PiZZ individuals compared with the general Swedish population.

Methods: Data on 1,561 PiZZ individuals from the Swedish National AAT Deficiency Register, prospectively followed from 1991 to 2014, were analyzed. Causes of death according to the Swedish National Causes of Death Register for the study group were compared with those for the general Swedish population matched for age, sex, and calendar year, with the excess mortality expressed as standardized mortality ratios (SMRs) with 95\% confidence intervals (CIs).

Results: There were 524 deaths during the follow-up period. PiZZ individuals had excess allcause mortality compared with the Swedish general population (SMR 3.6, 95\% CI 3.3-3.9). SMR for ischemic heart disease (IHD) was 0.5 (95\% CI 0.3-0.8) and was similar for never and ever-smokers, and in males and females. SMR for lung cancer was 0.9 (95\% CI 0.4-1.7). PiZZ individuals had increased mortality compared with the general population for the following diseases: respiratory disease, SMR 48.4 (95\% CI 43.0-54.5); primary liver carcinoma, SMR 90.0 (95\% CI 59.3-130.9); complicated colon diverticulitis, SMR 20.8 (95\% CI 6.7-48.6); and pulmonary embolism, SMR 6.9 (95\% CI 3.3-12.7).

Conclusion: PiZZ individuals had a reduced mortality risk of IHD. Mortality due to respiratory, hepatic disease, diverticulitis, and pulmonary embolism was markedly increased compared with the age- and sex-matched Swedish population.

Keywords: alpha 1-antitrypsin deficiency, cause-specific mortality, ischemic heart disease, pulmonary embolism, standardized mortality ratio

\section{Introduction}

Severe alpha 1-antitrypsin (AAT) deficiency (PiZZ) is an autosomal, codominant, hereditary disorder characterized by low AAT levels in the serum and lungs. The main function of AAT is to inhibit neutrophil elastase and other serine proteases, including proteinase- 3 and plasmin activator. ${ }^{1}$ This genetic defect results in the polymerization of the AAT molecule in the hepatocytes, leading to an accumulation of the Z-protein within the hepatocytes and a decreased release into the circulation. AAT deficiency contributes to protein degradation and increased inflammation because of proinflammatory effects of polymerized AAT and the loss of anti-inflammatory and antiproteolytic functions. ${ }^{2}$ In Sweden, the prevalence of AAT deficiency is $1 / 1,600$, a figure firmly established by a nationwide screening program of all 200,000 newborns over the period 1972-1974, when 127 homozygotes were identified. ${ }^{3}$ 
Major causes of disability and death associated with severe AAT deficiency are early-onset panacinar emphysema and liver disease, which typically presents as cholestasis in infancy, and cirrhosis and primary liver carcinoma (PLC) in adulthood. ${ }^{1}$ Association with systemic vasculitis (antineutrophil cytoplasmic antibody positive), necrotizing panniculitis, and a variety of different inflammatory and neoplastic diseases have also been reported. ${ }^{1,4,5}$

Previously published mortality studies have shown that severe AAT deficiency leads to reduced life expectancy and that respiratory failure and liver disease are the most common causes of death. ${ }^{6-8}$ Very little is known about mortality due to other diseases, such as cardiovascular disease (CVD), inflammatory diseases, and cancer, except for PLC that is a well-known risk in PiZZ individuals. ${ }^{9}$ Increased risk of lung cancer has also been described in carriers of the AATdeficient $\mathrm{Z}$ and $\mathrm{S}$ alleles. ${ }^{10}$

The Swedish National Register of individuals with severe AAT deficiency, PiZZ, has comprehensive physiological data and documents comorbidities by regular follow-ups every 2 years. ${ }^{11}$ Since 1991, all adult individuals with established severe AAT deficiency (PiZZ) in Sweden have been invited to participate in the register. After inclusion, the patients are prospectively followed up every 2 years by their attending physician, and the results are reported to the register.

The aim of this study was to analyze all-cause mortality and disease-specific mortality in the PiZZ individuals included in the Swedish National AAT Deficiency Register, with particular reference to ischemic heart disease (IHD), pulmonary embolism, lung cancer, and other diseases.

\section{Methods}

\section{Study population}

We analyzed data from the Swedish National AAT Deficiency Register, which has been detailed elsewhere. ${ }^{11}$ The study population included all PiZZ individuals included in the Swedish National AAT Deficiency Register since 1991. The inclusion criteria in the register are the PiZZ phenotype diagnosed by isoelectric focusing and age 18 years or older. The register is approved by the Ethical Review Board, Lund University, Sweden, and by the Swedish Data Inspection Board. The ethical approval includes analysis of mortality data. All study participants have provided written informed consent.

\section{Data collection}

Data on respiratory symptoms, diagnoses, spirometry, and smoking habits were reported by the attending physician to the Swedish National AAT Deficiency Register via a questionnaire. ${ }^{11}$ Lung function tests were performed at the local hospitals. The results of the lung function tests are expressed as the percentage of predicted values according to European reference tables. ${ }^{12}$ The results of the first spirometry at inclusion were analyzed.

Vital status and causes of death were obtained from the National Register of Causes of Death up to April 2014. The underlying cause of death was coded according to the ninth (before 1996) and the tenth revisions of the WHO International Classification of Diseases (ICD) ${ }^{13,14}$ ICD codes were grouped (ICD-9, ICD-10) as respiratory diseases (460-519×, 786; J00-J99, R04-R06), CVD (390-459×, 785; I00-I99, R00, R01), IHD (410-414; I20-I25), heart failure (425, 428; I42, I50, I51.7), stroke (430-438; I60-I69), aortic aneurysm (441; I71), venous thromboembolism $(415,451 ;$ I26, I80), digestive organ diseases (520-579×, 787; K00-K93), pneumonia (481-486; J13-J18), lung cancer (162; C34), colon cancer (153; C18), and other cancers (140-239×; C00-D48).

\section{Statistical analyses}

Baseline data were tabulated using frequencies and percentages for categorical variables, mean with standard deviation (SD), and median with range or interquartile range (IQR) for continuous variables with normal and skewed distribution, respectively. Comparisons of continuous variables with normal distribution were analyzed using analysis of variance (ANOVA). The period of follow-up was from the date of inclusion in the register to the date of death or up to April 1, 2014.

Excess mortality compared to the Swedish general population was calculated as standardized mortality ratios (SMRs), with respect to age and calendar year. The SMR is defined as the ratio of the observed deaths in the study population to the expected number of deaths. The expected number of deaths was calculated using the age-, sex-, and calendar year-specific mortality rates of the Swedish general population, obtained from the Swedish National Board of Health and Welfare. Age and calendar year were stratified in 5-year bands. SMRs were calculated for overall and cause-specific mortality for all patients, and separately for males/females and smokers/never-smokers. SMRs were expressed with 95\% confidence intervals (CIs) calculated from the Poisson distribution. Statistical significance was defined as a two-sided $P<0.05$. Statistical analyses were performed with the Statistical Package for the Social Sciences (SPSS), version 22.0 (IMB Corporation, Armonk, NY, USA).

\section{Results}

A total of 1,561 PiZZ individuals (49\% males) were included in the study. Baseline characteristics are shown in Table 1. 
Table I Demographic data of the PiZZ individuals at inclusion in the Swedish AAT Deficiency Register

\begin{tabular}{ll}
\hline Characteristics & $\mathbf{N}=\mathbf{I}, \mathbf{5 6} \mathbf{I}$ \\
\hline Never-smoker, $\mathrm{n}(\%)$ & $717(46)$ \\
Mean (SD) age at diagnosis (years) & $4 \mathrm{I}(2 \mathrm{I})$ \\
Mean (SD) age at inclusion (years) & $47(\mathrm{I7})$ \\
Mode of identification & \\
$\quad$ Respiratory, $\mathrm{n}(\%)$ & $670(43)$ \\
Liver disease, $\mathrm{n}(\%)$ & $\mathrm{III}(7)$ \\
Screening, $\mathrm{n}(\%)$ & $367(24)$ \\
Other, $\mathrm{n}(\%)$ & $413(26)$ \\
Mean (SD) FEV,$(\%$ predicted) & $74(33)$ \\
Mean (SD) FVC (\% predicted) & $94(24)$ \\
Mean (SD) FEV, /FVC ratio & $0.64(22)$ \\
Mean (range) number of pack-years & $14(0 . \mathrm{I}-\mathrm{II}$ I6) \\
Deaths, n (\%) & $524(34)$ \\
Mean age (SD) at death & $68(\mathrm{I} 2)$ \\
\hline Abbreviations: PiZZ, severe alpha I-antitrypsin deficiency; AAT, alpha I-antitrypsin; & \\
FEV, forced expiratory volume in I second; FVC, forced vital capacity; SD, standard \\
deviation.
\end{tabular}

Forty-six percent of the study population was never-smokers and $57 \%$ were identified for reasons other than respiratory symptoms. Females had smoked significantly less than males, with a mean (range) of $13(0.1-77)$ and $16(0.2-116)$ packyears, respectively $(P=0.003)$. During follow-up, 86 patients underwent lung transplantation. Lung function tests were available for 1,535 individuals (98\%) at inclusion, of whom 792 (52\%) fulfilled the criteria for COPD with a forced expiratory volume in 1 second $\left(\mathrm{FEV}_{1}\right)$ /forced vital capacity (FVC) ratio $<0.70$.

\section{Overall mortality}

The median follow-up period was 12 years $(\mathrm{IQR}=13)$ with a total of 18,881 person-years at risk of death. No patient was lost to follow-up. A total of 524 patients (277 males) died during the study. The SMR for PiZZ individuals was higher than that for the matched Swedish population (3.6, 95\% CI 3.3-3.9).

\section{Cause-specific mortality}

The causes of death were available for all decedents. The main causes of death were COPD and its complications such as respiratory failure and infections $(\mathrm{n}=281,54 \%)$, liver diseases $(n=74,14 \%), C V D(n=76,15 \%)$, and cancer $(\mathrm{n}=87,17 \%)$.

\section{Excess mortality}

PiZZ patients had significantly increased mortality due to respiratory and hepatic diseases, pulmonary embolism, and colon diverticulitis compared with the general Swedish population, as shown in Table 2. In contrast, they had a
Table 2 Cause-specific mortality in severe alpha I-antitrypsin deficiency

\begin{tabular}{lll}
\hline Disease types & Observed/expected & SMR (95\% CI) \\
\hline Overall SMR & $524 / 147$ & $3.6(3.3-3.9)$ \\
COPD & $281 / 5.8$ & $48.4(43.0-54.5)$ \\
Hepatic & & \\
$\quad$ Liver failure/complications & $44 / 0.9$ & $47.8(35.8-64.2)$ \\
$\quad$ Primary liver carcinoma & $27 / 0.3$ & $90.0(59.3-130.9)$ \\
$\quad$ Cholangiocarcinoma & $3 / 0.2$ & $15.0(3.1-43.8)$ \\
Colon diverticulitis & $5 / 0.2$ & $20.8(6.7-48.6)$ \\
Cardiovascular disease & $76 / 82.2$ & $0.9(0.7-1.2)$ \\
Ischemic heart disease & $22 / 42.1$ & $0.5(0.3-0.8)$ \\
Heart failure & $18 / 4.5$ & $4.0(2.4-6.3)$ \\
Pulmonary embolism & $10 / 1.4$ & $6.9(3.3-12.7)$ \\
Aorta dissection & $3 / 2.9$ & $1.0(0.2-3.0)$ \\
Stroke & $19 / 17.8$ & $1.1(0.6-1.7)$ \\
All cancer & $87 / 58.6$ & $1.5(1.2-1.8)$ \\
Lung cancer & $9 / 10.3$ & $0.9(0.4-1.7)$ \\
Colon cancer & $8 / 5.3$ & $I .5(0.6-3.0)$ \\
Breast cancer & $6 / 4.4$ & $1.4(0.5-3.0)$ \\
\hline
\end{tabular}

Abbreviations: SMR, standardized mortality ratio; $\mathrm{Cl}$, confidence interval.

reduced risk of mortality due to IHD, and no increased mortality risk due to CVD as a whole. However, mortality due to heart failure was significantly increased. Of the 18 patients who died of heart failure, eleven had COPD, one had pulmonary fibrosis, and one had Marfan's syndrome. Complicated colon diverticulitis with peritonitis was the cause of death of five patients; none of these patients had known inflammatory bowel disease.

\section{Sex-related differences in mortality}

The mean age at death was lower in males (67 years, SD 11 years) compared with females (69 years, SD 12 years). SMR for males was $3.6(95 \%$ CI 3.2-4.0) and 3.5 (95\% CI 3.1-4.0) for females.

An excess mortality for PLC was observed in both males and females. Twenty males died of PLC compared with an expected 0.2 deaths, giving an SMR of 100 (95\% CI 61-154), and seven females compared with an expected 0.09 deaths, giving an SMR of 77.8 (95\% CI 31.3-160.2). There was no difference in mortality due to respiratory diseases, liver failure, and CVD between males and females.

\section{Smoking-related differences in mortality}

During the follow-up period, 350 ever-smokers and 174 never-smokers died. The mean age at death was lower in eversmokers compared with never-smokers (65 years, SD 11 and 74 years, SD 11, respectively). The SMR for ever-smokers was higher than for never-smokers $(4.6$ [95\% CI 4.1-5.1] and 2.5 [95\% CI 2.1-2.9], respectively). Mortality due to respiratory diseases was also higher in ever-smokers compared with never-smokers (Table 3). No difference was found in 
Table 3 Cause-specific mortality in ever-smokers and never-smokers with severe alpha I-antitrypsin deficiency

\begin{tabular}{|c|c|c|c|c|}
\hline & \multicolumn{2}{|l|}{ Ever-smokers } & \multicolumn{2}{|l|}{ Never-smokers } \\
\hline & Observed/expected & SMR $(95 \% \mathrm{Cl})$ & Observed/expected & SMR $(95 \% \mathrm{CI})$ \\
\hline COPD & $214 / 3.0$ & $71.3(62.1-81.6)$ & $67 / 2.8$ & $24.0(\mid 8.5-30.4)$ \\
\hline \multicolumn{5}{|l|}{ Hepatic } \\
\hline Liver cirrhosis/complications & $25 / 0.53$ & $47.2(30.5-69.6)$ & $19 / 0.39$ & $48.7(29.3-76.1)$ \\
\hline Primary liver carcinoma & $12 / 0.17$ & $70.6(36.5-123.3)$ & $15 / 0.12$ & I25.0 (70.0-206.2) \\
\hline Cholangiocarcinoma & I/0.II & $9.1(0.2-50.7)$ & $2 / 0.86$ & $2.3(0.3-8.4)$ \\
\hline Cardiovascular disease & $40 / 40.2$ & $1.0(0.7-1.4)$ & $36 / 41.1$ & $0.9(0.6-1.2)$ \\
\hline Ischemic heart disease & $13 / 21.6$ & $0.6(0.3-1.0)$ & $9 / 20.0$ & $0.4(0.2-0.8)$ \\
\hline Heart failure & $8 / 1.7$ & $4.7(2.0-9.3)$ & $10 / 2.6$ & $3.8(1.8-7.1)$ \\
\hline Pulmonary embolism & $6 / 0.74$ & $8.1(3.0-17.7)$ & $4 / 0.70$ & $5.7(1.6-14.6)$ \\
\hline Stroke & $9 / 8.2$ & I.I (0.5-2.I) & $10 / 9.3$ & I.I $(0.5-2.0)$ \\
\hline Lung cancer & $6 / 6.2$ & I.0 (0.36-2.I) & $3 / 4.1$ & $0.7(0.2-2.1)$ \\
\hline
\end{tabular}

Abbreviations: SMR, standardized mortality ratio; $\mathrm{Cl}$, confidence interval.

mortality due to IHD, pulmonary embolism, and lung cancer between ever-smokers and never-smokers. Mortality due to PLC tended to be higher in the never-smokers than in the ever-smokers.

\section{Lung function-related differences in mortality}

Mortality due to respiratory diseases was five times higher in individuals with $\mathrm{COPD}\left(\mathrm{FEV}_{1} / \mathrm{FVC}\right.$ ratio $\left.<0.70\right)$ at inclusion compared with those with normal lung function (Table 4). Mortality due to IHD was less than in the general population in both groups. An excess mortality due to heart failure was found in AAT-deficient individuals irrespective of lung function.

\section{Cause-specific mortality in individuals identified by screening}

Individuals who were identified by screening $(n=367)$ had significantly higher overall mortality compared with the general Swedish population, SMR 2.2 (95\% CI 1.6-3.0). They had significant excess mortality from the following diseases: respiratory disease, SMR 27.1 (95\% CI 16.3-42.3); PLC, SMR 42 (95\% CI 5.1-151.0); liver cirrhosis, SMR 14.5 (95\% CI 1.8-52.3); and heart failure, SMR 14.5 (95\%
CI 4.7-33.8). Mortality due to CVD, all cancer, and lung cancer was similar to that in general population, SMR 1.3 (95\% CI 0.63-2.3), SMR 0.9 (95\% CI 0.3-1.8), and SMR 0.7 (95\% CI 0.02-3.7), respectively.

\section{Discussion}

This study demonstrates that individuals with severe AAT deficiency have a reduced mortality due to IHD compared with the Swedish general population matched for age, sex, and calendar year. This finding cannot be explained by differences in smoking habits, as a similar reduction was seen in both smokers and nonsmokers. We also found that the mortality rate due to CVD as a whole was similar to that in the general population.

To our knowledge, this is the first study that analyzes cause-specific mortality in individuals with severe AAT deficiency. Previous mortality studies have compared mortality either between never- and ever-smoking PiZZ individuals or between PiZZ individuals and the general population. ${ }^{6-8}$ These studies have demonstrated an increased mortality risk in PiZZ individuals compared with the general population, and a significantly reduced survival in PiZZ smokers compared with never-smokers. Emphysema and liver cirrhosis

Table 4 Cause-specific mortality by airflow limitation in severe alpha I-antitrypsin deficiency

\begin{tabular}{|c|c|c|c|c|}
\hline & \multicolumn{2}{|l|}{ FEV $_{\mathrm{I}} / \mathrm{FVC}$ ratio $\geq \mathbf{0 . 7 0}$} & \multicolumn{2}{|c|}{ FEV $_{\text {I }}$ FVC ratio $<0.70$} \\
\hline & Observed/expected & SMR (95\% Cl) & Observed/expected & $\operatorname{SMR}(95 \% \mathrm{CI})$ \\
\hline COPD & $26 / 1.8$ & $\mid 4.4(9.4-2 \mid .2)$ & $253 / 3.9$ & $64.9(57.1-73.4)$ \\
\hline Cardiovascular disease & $29 / 25.0$ & $1.2(0.8-1.7)$ & $47 / 54.6$ & $0.9(0.6-1.1)$ \\
\hline Ischemic heart disease & $5 / 12.2$ & $0.4(0.1-0.9)$ & $17 / 28.8$ & $0.6(0.3-0.9)$ \\
\hline Heart failure & $7 / 1.5$ & $4.7(1.9-9.6)$ & $\mathrm{I} \mathrm{I} / 2.8$ & $3.9(2.0-7.0)$ \\
\hline Pulmonary embolism & $2 / 0.5$ & $4.0(0.5-14.4)$ & $8 / 0.94$ & $8.5(3.7-16.8)$ \\
\hline Lung cancer & $4 / 3.2$ & $1.3(0.3-3.2)$ & $5 / 6.9$ & $0.7(0.2-1.7)$ \\
\hline
\end{tabular}

Abbreviations: $\mathrm{FEV}_{1}$, forced expiratory volume in I second; $\mathrm{FVC}$, forced vital capacity; SMR, standardized mortality ratio; $\mathrm{Cl}$, confidence interval. 
have been the predominant causes of death in both ever- and never-smokers. Mortality due to CVD was not analyzed in these studies.

One possible mechanism for the reduced mortality from IHD is that PiZZ individuals may have lower blood pressure than healthy controls, as reported by Dahl et al. ${ }^{15}$ Hypertension being a major risk factor for both CVD and IHD, a lower level of blood pressure in AAT-deficient individuals than in the general population could reduce the risk of CVD and IHD. Dichtl et $\mathrm{al}^{16}$ found that AAT deficiency leads to fewer cleaved fragments of AAT in atherosclerotic plaques and thereby reduces atherosclerotic inflammation and risk of IHD. An interesting hypothesis is that a low serum concentration of AAT or the $\mathrm{Z}$ mutation of the AAT molecule could provide biochemical or genetic protection against IHD. However, Duckers et $\mathrm{al}^{17}$ reported increased aortic stiffness in a small group of PiZZ individuals with COPD in comparison with age- and sex-matched controls with normal lung function, indicating increased cardiovascular risk. No patients with usual COPD were included in the study, and it therefore remains unclear whether the increased aortic stiffness was related to AAT deficiency or COPD per se. In our study, $48 \%$ of PiZZ individuals had normal lung function. We analyzed cause-specific mortality, and therefore it is difficult to compare our results with other studies that analyze risk of disease or clinical signs of diseases.

Interestingly, the risk of death from pulmonary embolism was markedly increased - seven times higher than in the general population. This is a new finding and has previously not been reported in mortality studies. ${ }^{7,8}$ In our previously published study, six (2\%) of 302 deaths were caused by pulmonary embolism. ${ }^{6} \mathrm{~A}$ few case-report studies have suggested that AAT deficiency may be associated with an increased risk of venous thromboembolism. ${ }^{18-20}$ There are two possible mechanisms for increased thrombophilia in AAT deficiency. An unopposed proteolytic activity of plasminogen activator could cause the activation of the coagulation cascade. Additionally, unopposed proteinase-3 activity may damage and/or activate endothelial cells, resulting in a prothrombotic state. ${ }^{20}$ Increased mortality from heart failure is easier to explain, because COPD and respiratory failure are common causes of right heart failure.

Because previously published studies have shown that patients with COPD have increased mortality due to CVD, ${ }^{21}$ we also analyzed SMR stratified by lung function. We still did not find any increased mortality due to CVD in the individuals with reduced lung function $\left(\mathrm{FEV}_{1} / \mathrm{FVC}\right.$ ratio $<0.70)$ compared with those with normal lung function.
Simultaneously, the decreased mortality due to IHD and the increased mortality due to heart failure and pulmonary embolism remained significant in both the individuals with COPD and those with normal lung function. We found that mortality due to respiratory diseases was 14 times higher in AAT-deficient individuals with normal lung function at baseline than in the general population. Furthermore, AATdeficient individuals with COPD at inclusion had a fivefold higher risk of mortality due to respiratory diseases compared with those with normal lung function.

As expected, we found greatly increased mortality in PLC. Increased mortality due to cancer, overall, was driven by increased mortality due to PLC, while mortality due to lung, breast, or colon cancer was not increased. Previously published studies have indicated that AAT deficiency carriers have increased risk of lung and bladder cancer, and malignant lymphoma. Yang et $\mathrm{al}^{10}$ have reported, in a case-control study, an increased risk of lung cancer in carriers of AAT-deficient $\mathrm{Z}$ and $\mathrm{S}$ alleles compared with noncarriers. They concluded that the risk was caused by an imbalance between AAT and neutrophil elastase. It is possible that the burden of smoking is lower among the AAT-deficient patients than among the patients with usual COPD. Because no smoking data are available for the general population, this hypothesis cannot be tested in this study.

An unexpected finding was the increased mortality due to complicated diverticulitis. Stoller et $\mathrm{al}^{7}$ have previously reported that diverticulitis was the underlying cause of death in 3\% of the 120 decedents with severe AAT deficiency in the National Heart, Lung and Blood Institute Register. In our study, five of the 524 decedents (1\%) died of diverticulitis, which was significantly higher than the expected 0.24 deaths in the Swedish general population.

\section{Strengths and limitations of this study}

One of the most important strengths of our study is that all data are collected from a well-established national register of PiZZ individuals with a correct diagnosis verified by isoelectric focusing. In Sweden, the detection rate of severe AAT deficiency is relatively high (30\%). The majority of individuals in the register had been identified in investigations for reasons other than respiratory symptoms, and 24\% were identified by family or population screening. Due to these facts, a large number of the study population (48\%) had normal lung function at inclusion. The follow-up period was long, from 1991 to 2014.

Another important strength in our study is that it is based on the Swedish system of personal identification number, 
which implies that the vital status was available for all subjects. In the national mortality statistics, the causes of death are recorded for all deceased individuals in Sweden. Using a relative mortality model and the national mortality statistics made it possible to describe the excess causespecific mortality among the PiZZ individuals compared with the matched general population with complete follow-up.

There are some limitations in our study. First, the mortality estimates are based on data from death certificates. The cause-specific estimates could be affected by changes over time in diagnostics as well as changes in coding and classification of the causes of death by physicians and in the Swedish Causes of Death Register. It is unlikely that the change from ICD-9 to ICD-10 in 1997 has affected the reliability of the results, according to analysis performed by the Swedish National Board of Health and Welfare. ${ }^{22}$ The possible bias is unlikely to substantially affect the internal or external validity of the present study. Furthermore, we cannot completely exclude that observed differences (or lack thereof) between the analyzed patient categories (by sex, smoking, and lung function) may be caused by differences in person-year distribution with respect to age and/or calendar year. Third limitation is that no individual data on smoking habits in the Swedish general population are available.

\section{Conclusion}

We conclude that PiZZ individuals had a reduced mortality risk of IHD. Further studies of CVD and cancer in PiZZ individuals compared with controls with known smoking habits are needed.

\section{Acknowledgments}

The authors wish to thank Isabella Björk for secretarial assistance. They also thank all the Swedish physicians who report data to the Swedish AAT deficiency registry. HAT was supported by unrestricted grants from the Swedish Society of Medicine. ME was supported by unrestricted grants from the Swedish Society of Medicine and the Swedish Heart-Lung Foundation. EP was supported by unrestricted grants from the Swedish Heart-Lung Foundation.

\section{Disclosure}

The authors report no conflicts of interest in this work.

\section{References}

1. American Thoracic Society/European Respiratory Society Statement. Standards for the diagnosis and management of individuals with alpha-1 antitrypsin deficiency. Am J Respir Crit Care Med. 2003;168(7): 818-900.
2. Ekeowa UI, Gooptu B, Belorgey D, et al. Alpha1-antitrypsin deficiency, chronic obstructive pulmonary disease and the serpinopathies. Clin Sci. 2009; $116(12): 837-850$.

3. Sveger $\mathrm{T}$. Liver disease in alpha1-antitrypsin deficiency detected by screening of 200,000 infants. N Engl J Med.1976;294(24):1316-1321.

4. Segelmark M, Elzouki A-N, Wieslander J, Eriksson S. The PiZZ gene of alpha 1-antitrypsin as a determinant of outcome in PR3-ANCA-positive vasculitis. Kidney Int. 1995;48(3):844-850.

5. Smith KC, Su WP, Pittelkow MR, Winkelmann RK. Clinical and pathologic correlations in 96 patients with panniculitis, including 15 patients with deficient levels of alpha 1-antitrypsin. J Am Acad Dermatol. 1989; 21(6):1192-1196.

6. Tanash HA, Nilsson PM, Nilsson JA, Piitulainen E. Survival in severe alpha-1-antitrypsin deficiency (PiZZ). Respir Res. 2010;11:44.

7. Stoller JK, Tomashefski J Jr, Crystal RG, et al. Mortality in individuals with severe deficiency of alpha1-antitrypsin: findings from the National Heart, Lung, and Blood Institute Registry. Chest. 2005;127(4): 1196-1204.

8. Seersholm N, Kok-Jensen A, Dirksen A. Survival of patients with severe alpha 1-antitrypsin deficiency with special reference to non-index cases. Thorax. 1994;49(11):695-698.

9. Eriksson S, Carlson J, Velez R. Risk of cirrhosis and primary liver cancer in alpha 1-antitrypsin deficiency. N Engl J Med. 1986;314(12): 736-739.

10. Yang P, Bamlet WR, Sun Z, et al. Alpha1-antitrypsin and neutrophil elastase imbalance and lung cancer risk. Chest. 2005;128(1):445-452.

11. Piitulainen E, Tanash HA. The clinical profile of subjects included in the Swedish National Register on individuals with severe alpha 1-antitrypsin deficiency. COPD. 2015;12(Suppl 1):36-41.

12. Quanjer P. Standardized lung function testing. Report working party. Bull Eur Physiopathol Respir. 1983;19(Suppl 5):1-95.

13. World Health Organization. Manual of the International Classification of Diseases, Injuries and Causes of Death. 9th revision. Vol 1. Geneva, Switzerland: WHO; 1978.

14. World Health Organization. Manual of the International Statistical Classification of Diseases and Health Related Problems. 10th revision. Vol 1. Geneva, Switzerland: WHO; 1992.

15. Dahl M, Tybjaerg-Hansen A, Nordestgaard BG. Risk of ischemic heart and ischemic cerebrovascular disease is not increased in $\mathrm{S}, \mathrm{Z}$, and 11478A alpha1-antitrypsin carriers of the Copenhagen City Heart Study. Arterioscler Thromb Vasc Biol. 2003;23(11):644-649.

16. Dichtl W, Moraga F, Ares MPS, et al. The carboxyl-terminal fragment of 1-antitrypsin is present in atherosclerotic plaques and regulates inflammatory transcription factors in primary human monocytes. Mol Cell Biol Res Commun. 2000;4(1):50-61.

17. Duckers JM, Shale DJ, Stockley RA, et al. Cardiovascular and musculskeletal co-morbidities in patients with alpha 1 antitrypsin deficiency. Respir Res. 2010;11:173

18. Elsensohn AN, Curtis JA, Secrest AM, et al. Alpha-1-antitrypsin deficiency panniculitis presenting with severe anasarca, pulmonary embolus, and hypogammaglobulinemia. Br J Dermatol. 2015;173(1):289-291.

19. Gupta R, Sridhara S, Wood JA. A rare case of alpha 1-antitrypsin deficiency associated with hypogammaglobulinemia and recurrent pulmonary thrombosis. Ann Thorac Med. 2014;9(1):39-41

20. Milger K, Holdt LM, Teupser D, Huber RM, Behr J, Kneidinger N. Identification of a novel SERPINA-1 mutation causing alpha-1 antitrypsin deficiency in a patient with severe bronchiectasis and pulmonary embolism. COPD. 2015;10:891-897.

21. Sin DD, Wu L, Man SF. The relationship between reduced lung function and cardiovascular mortality: a population-based study and a systematic review of the literature. Chest. 2005;127(6):1952-1959.

22. Johansson L. Mortality bridge coding ICD9/ICD10: preliminary results from a Statistics Sweden study (WHO/GPE/ICD/C/00.66). In: Meeting of heads of WHO collaborating centres for the classification of diseases; October 15-21, 2000; Rio de Janeiro, Brazil. Publication no. WHO/ GPE/ICD/C/00.71. Geneva, Switzerland: World Health Organization; 2000. Available from: http://www.who.int/classifications/ network/en/ report2000.pdf. Accessed October 1, 2000. 
International Journal of COPD

\section{Publish your work in this journal}

The International Journal of COPD is an international, peer-reviewed journal of therapeutics and pharmacology focusing on concise rapid reporting of clinical studies and reviews in COPD. Special focus is given to the pathophysiological processes underlying the disease, intervention programs, patient focused education, and self management protocols.

This journal is indexed on PubMed Central, MedLine and CAS. The manuscript management system is completely online and includes a very quick and fair peer-review system, which is all easy to use. Visit http://www.dovepress.com/testimonials.php to read real quotes from published authors 